\title{
Localization of Subsurface Structures for Site-Specific Cryo-FIB Lift-Out Preparation of Solid-Liquid Interfaces
}

\author{
Michael J. Zachman ${ }^{1,2}$ and Lena F. Kourkoutis ${ }^{1,2}$ \\ 1. School of Applied and Engineering Physics, Cornell University, Ithaca, NY 14853, USA. \\ 2. Kavli Institute for Nanoscale Science, Cornell University, Ithaca, NY 14853, USA.
}

While proof of concept demonstrations of cryo-FIB lift-out have recently been discussed [1-5], a method of localizing subsurface structures that can guide the TEM sample preparation of non-biological systems is thus far absent. Analogous to FIB lift-out routinely used to prepare electron transparent TEM samples from bulk materials, cryo-FIB lift-out promises the ability to extract structures in their near-native environment from large intact soft, liquid, or hard-soft materials. However, the limited throughput of the technique and small volume of material prepared require localization of buried structures of interest prior to milling. This issue was recently addressed in biology, where fluorescent labeling of molecules and cryo-fluorescence light microscopy (cryo-FLM) can be used to localize structures prior to cryo-FIB preparation [5]. Cryo-FIB and cryo-FLM images can then be correlated to identify regions of interest for milling. This technique cannot be applied to samples that lack fluorescent labels, however, so an alternative localization technique is needed for these systems.

We present here a technique for localizing subsurface structures in intact hard-soft materials systems, at optimal depths for cryo-FIB lift-out. Both backscattered electron (BSE) imaging and energy dispersive $\mathrm{x}$-ray spectroscopy (EDX) mapping can probe structures below the specimen's surface. A model system of iron oxide particles grown in a silica hydrogel was used to evaluate the capabilities of these signals. Electron scattering calculations revealed the characteristic Fe-K $\alpha$ x-ray generation depth exceeds the range of BSE, as depicted in Figure 1a. Monte Carlo simulations were then used to directly compare BSE and EDX signals from an appropriately sized iron oxide particle embedded at various depths in water under a $10 \mathrm{~nm}$ platinum layer, scanned with a $30 \mathrm{kV}$ electron beam, as shown in Figure $1 \mathrm{~b}$. These results confirm, while BSE imaging can identify heavy particles near the surface, EDX mapping can readily localize particles with element specificity to depths beyond the typical size of a lamella. These results are consistent with experimental observations in the cryo-FIB, shown in Figure 2. EDX was used to localize individual iron oxide particles at the proper depth in our hydrogel system and guide precision milling. Thin and homogeneous lamellas were then prepared for cryo-STEM, as shown in Figure 2c, enabling high-resolution cryo-STEM imaging to be performed on the particles, as in Figure 2f. EDX has thus proven to be an effective tool for localizing subsurface structures in hard-soft materials for cryoFIB lift-out and subsequent high-resolution characterization by cryo-STEM. Consequently, use of this technique can dramatically increase the yield of desired structures in lamellas of appropriate samples, significantly increasing the effectiveness of cryo-FIB lift-out for a number of fields [6].

[1] S. Rubino et al., J. Struct. Biol. 180 (2012), p. 572-576.

[2] N. Antoniou et al., 38th International Symposium for Testing and Failure Analysis (2012), p. 399.

[3] C. Parmenter et al., Microsc. Microanal. 20 (Suppl 3) (2014), p. 1224.

[4] M. Zachman et al., Microsc. Microanal. 21(Supp 3) (2015), p. 2291.

[5] J. Mahamid et al., J. Struct. Biol. 192 (2015), p. 262.

[6] We thank Lara A. Estroff and Emily Asenath-Smith for providing the samples used in this work. This work was supported by the Packard Foundation, a KIC graduate fellowship, and made use of the CCMR Shared Facilities which are supported through the NSF MRSEC program (DMR-1120296). 

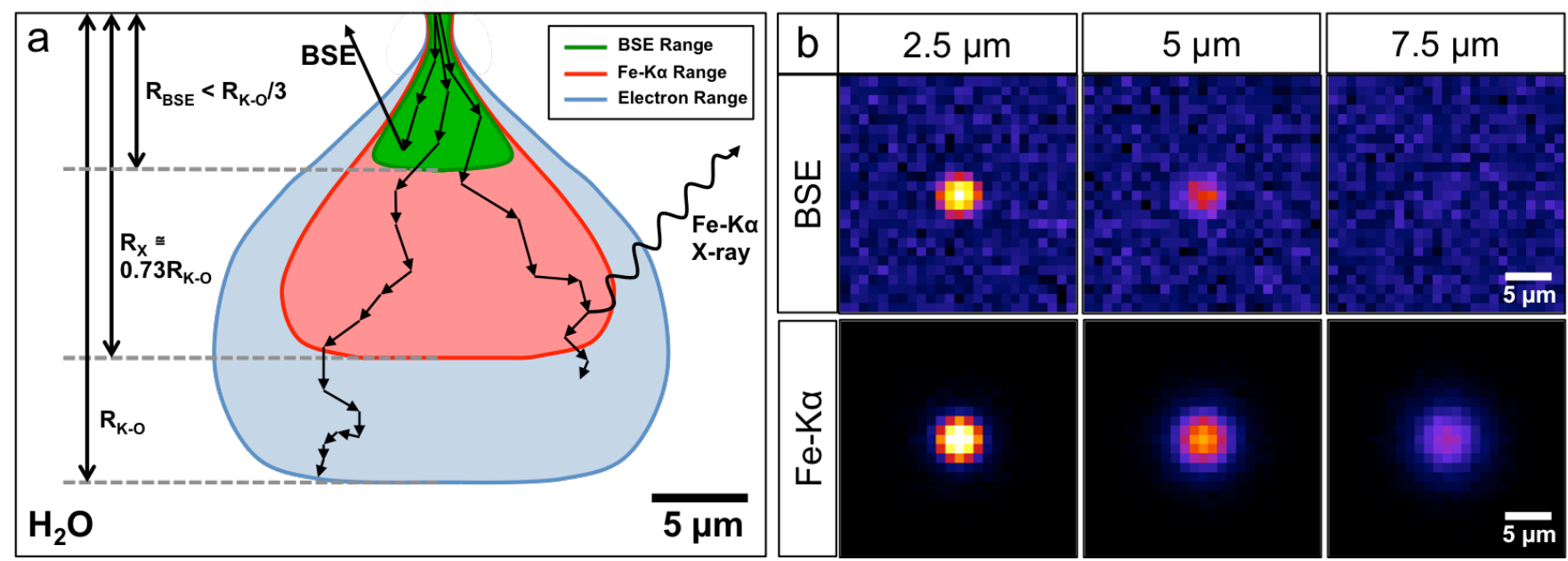

Figure 1. (a) Range of primary electrons and volumes probed by $\mathrm{Fe}-\mathrm{K} \alpha$ characteristic x-rays and backscattered electrons, calculated with a $30 \mathrm{kV}$ beam and a water sample. (b) Comparison of the subsurface structure localization capabilities of backscattered electrons and Fe-Ka X-rays by Monte Carlo simulations of a $2.5 \mu \mathrm{m}$ radius $\mathrm{Fe}_{2} \mathrm{O}_{3}$ particle in water, beneath a $10 \mathrm{~nm}$ platinum surface layer.
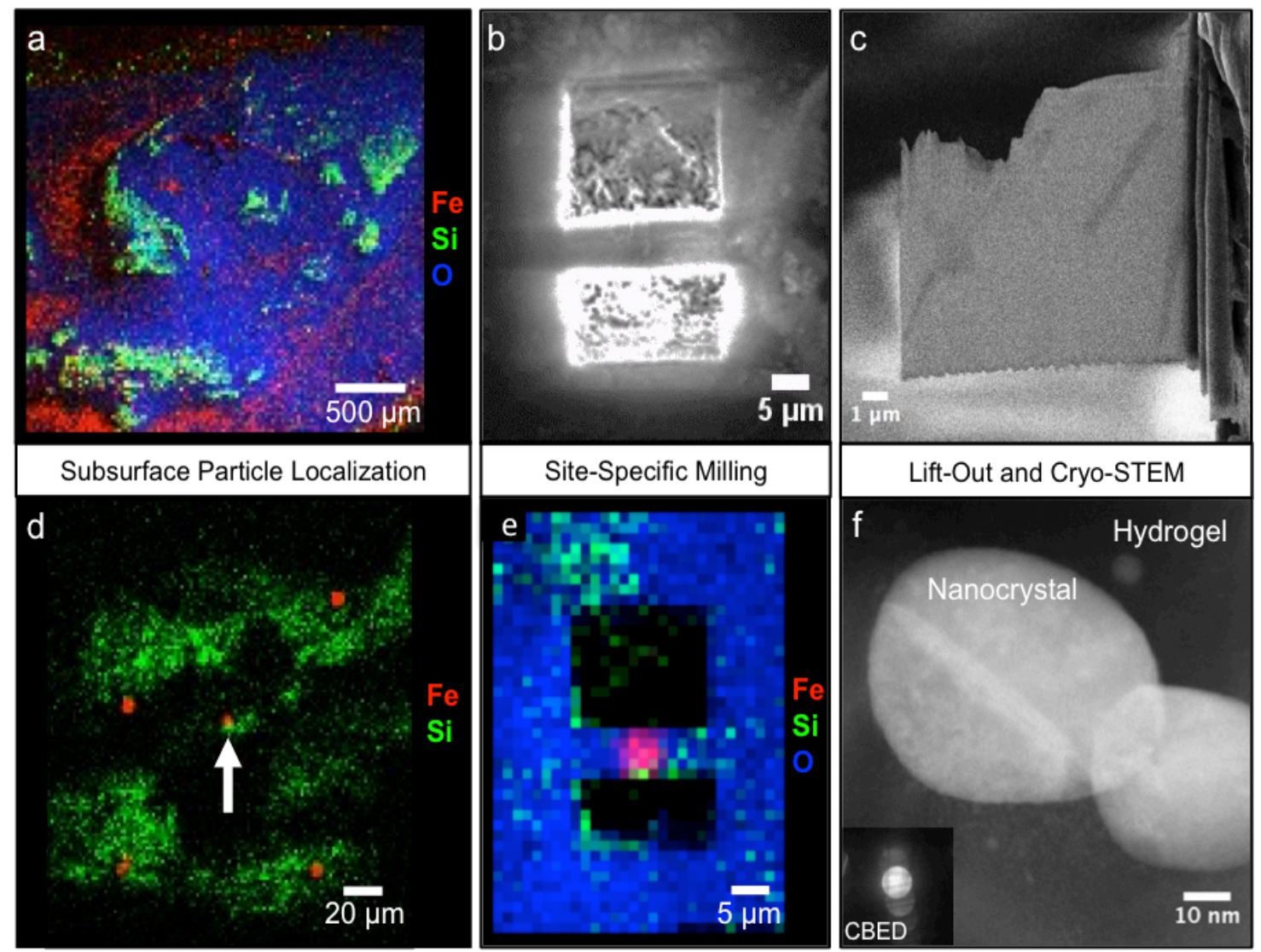

Figure 2. EDX allows regions of interest to be identified by mapping elemental distributions (a), as well as localization of individual structures at appropriate depths for cryo-FIB lift-out (d). While the electron signal reveals little about the contents of the lamella (b), EDX can be used for precise lift-out of appropriate structures (e). Cryo-FIB enables preparation of thin and homogeneous lamellas (c), allowing high-resolution cryo-STEM characterization of intact solid-liquid interfaces to be performed (f). 